\title{
Determination of the cavity mode in the water
}

\author{
Pavel Vikulin ${ }^{1}$ and Vera Vikulina ${ }^{{ }^{*}}$ \\ ${ }^{1}$ Moscow State University of Civil Engineering, Yaroslavskoye shosse, 26, Moscow, Russia, 129337
}

\begin{abstract}
Ultrasonic oscillations find their wide application in various sectors and industries: metallurgy, chemical and food industries, in engineering, in medicine. This is due to physical and chemical changes when exposed to the sound field. Cavitation in ultrasonic field causes the dispersion and emulsification of certain substances and promotes coagulation and degassing affects of crystallization and dissolution. It is known that ultrasonic vibrations also cause a variety of chemical transformations of substances such as oxidation, recovery, polymerization, and depolymerization. Researchers of cavitation action on the matter find the explanation of these phenomena: shock waves and, consequently, acoustic wind. The experiments were conducted in liquid environment (non-distilled water). The volume of the experimental sample amounted to $10 \mathrm{dm}^{3}$. Method of the magnetostriction was applied to receive ultrasonic vibration, the principle of which consists in transforming electrical oscillations into mechanical. The level of cavitation was checked in two stages to evaluate the cavitation mode according to the degree of erosion of the artificial barriers and to measure the intensity of cavitation noise in the volume.
\end{abstract}

\section{Introduction}

\subsection{Nature of ultrasound}

Ultrasound is elastic vibrations. The laws of non-linear acoustics can describe ultrasonic waves of high intensity. The distribution of ultrasonic waves in liquids is accompanied by acoustic flow, compression and rarefaction of the environment. Acoustic cavitation is among the important non-linear phenomena in ultrasonic field. Investigation of the influence of acoustic fields on substances is complicated due to various simultaneous processes that can influence each other in the ultrasonic field. Therefore, it is difficult to describe experimental results. At present, theoretical justification of physical methods is evaluated, in particular ultrasonic vibrations in water technologies. Application of ultrasonic field for the intensification of redox processes in the water environment, deposition of particulate impurities extends the scope of the possible use of this physical method $[1,2,3,4]$.

Sound as a physical phenomenon, is characterized by sound pressure, sound energy density, sound energy flux, the intensity level (power) of the sound. Fluid in a static state

\footnotetext{
${ }^{*}$ Corresponding author: nocviv@mgsu.ru
} 
does not have the shear viscosity and is not able to withstand and transmit any shear stresses. Therefore, longitudinal waves only can distribute in liquids and gases in which the direction of the tangential movements of particles coincides with the direction of wave propagation. The speed of distribution depends on the density of the medium $\rho$ and adiabatic compressibility $\beta c$ calculated by the formula:

$$
c=\sqrt{\frac{1}{\rho \cdot \beta_{c}}} .
$$

Physical nature of elastic waves distribution can be described by the equation:

$$
y=A \cdot \sin \omega \cdot t
$$

$A$ - offset amplitude; $\omega$ - cycle frequency; $t$ - time.

Acoustic vibrations in the environment creates additional pressure [5, 6, 7]. A sound wave passing through the fluid creates areas of compression and refraction, which change at every half period of the passing wave. When this occurs, the alternating pressure P, which can be determined by the formula:

$$
P=\sqrt{r \cdot c \cdot I \cdot 4,6 \cdot 10^{-3}}
$$

Sound pressure is variable and changes periodically. At this point the environment during the period, the pressure $\mathrm{P}$ changes from a maximum to zero and then increases again to a maximum value, and corresponds to the description of harmonic oscillations:

$$
P=P_{\max } \cdot \sin \omega \cdot t
$$

$P_{\max }-$ maximum sound pressure (pressure amplitude) defined by the formula:

$c$ - speed of sound.

$$
P_{\max }=\omega \cdot c \cdot r \cdot A
$$

Within the distribution of ultrasound in the environment, part of its energy is absorbed, heating of the environment. The absorption of acoustic energy happens due to the frequency of sound, viscosity, thermal conductivity and impedance of environment, i.e. the product of the density of the medium $\rho$ and the speed of sound. The connection between sound pressure and impedance expresses the oscillatory speed. [8, 9]

The value of the medium wave resistance is determined by the ratio of sound pressure in a running flat wave to the particle velocity of the medium $V$

$$
\frac{P}{V}=r \cdot c \text {. }
$$

Vibrational velocity and sound pressure do not depend on the frequency, the amplitude is inversely proportional, and the acceleration is directly proportional to the frequency.

For moderate acoustic fields acoustic pressure does not typically exceed one MPa, however, the pressure gradient, especially at high frequencies, can reach large values. Amplitude accelerations of fluid particles in a field of ultrasonic waves may exceed the acceleration of free fall by several orders. Such acceleration is achieved only in special ultracentrifuge taking into account that such values of acceleration change sign twice during the period ultrasonic waves may be considered as a very powerful and distinctive physical factor influencing the substance.

\subsection{Physical and chemical effects in the liquid medium under the action of ultrasonic cavitation}

Basic physical and chemical effects that occur in fluid under the action of the acoustic field, are caused mainly to nonlinear effects, the most important of which is cavitation $[10,11$, $12,13]$. One of the features of ultrasonic cavitation is that it is a unique and effective mechanism for the local concentration of a relatively low average energy of the acoustic field at very small volumes, resulting in extremely high energy densities. 
The detailed mechanism of this effect remains unclear. Because of a great theoretical and practical interest in the basic physical-chemical cavitation problems in the following will be given significant attention.

The impedance of the environment is a very important characteristic that determines the conditions of radiation, absorption of acoustic waves, their reflection, refraction, etc.

The particles of an elastic medium, in which ultrasonic waves are distributed oscillate and therefore possess kinetic and potential energy.

The amount of energy carried the sound vibrations for $1 \mathrm{sec}$ through an area of $1 \mathrm{~cm}^{2}$ perpendicular to the direction of propagation, describes sound intensity and is determined by the formula:

$E_{1}$ - density of energy.

$$
I=E_{1} \cdot c
$$

The sound energy density at each point changes with time. The average value of the energy density at a given point is determined by the formula:

$$
E_{1}=\frac{1}{2} \cdot A^{2} \cdot \omega^{2} \cdot \rho
$$

Transforming equation (7) and equation (8) we get the expression (9):

$$
I=\frac{1}{2} \cdot A^{2} \cdot \omega^{2} \cdot \rho \cdot c
$$

Within the distribution of sound waves in a liquid environment, the sound intensity I decreases with increasing distance from the radiation source according to the equation:

$$
I=I_{0} \cdot e^{2 \alpha X}
$$

$I_{0}$ - sound intensity if $X=0 ; \alpha$-adsorption coefficient.

The absorption coefficient that depends on the physical properties of a substance is a characteristic of the substance and depends on external conditions (temperature, pressure) and frequency.

Although the physical nature of ultrasound and the basic laws describing the distribution are the same as for sound waves of any frequency range, it has a number of specific features. These features are due to the relatively high frequencies and, accordingly, the smallness of the wavelengths $[14,15,16]$.

So, for high ultrasonic frequencies, the wave lengths:

$$
\begin{array}{cc}
3,4 \cdot 10^{-3}-3,4 \cdot 10^{-5} \mathrm{~cm} & \text { In the air, } \\
1,5 \cdot 10^{-2}-1,5 \cdot 10^{-4} \mathrm{~cm} & \text { In the water, } \\
5 \cdot 10^{-2}-5 \cdot 10^{-4} \mathrm{~cm} & \text { In metals. }
\end{array}
$$

Ultrasonic waves attenuate much faster than waves of low frequency sound range, as the ratio of the "classical" sound absorption is proportional to the square of the frequency.

In the low frequency area, the coefficient of relaxation absorption also increases in proportion to the square of the frequency, however at higher frequencies this increase stops and the absorption coefficient tends to a constant value.

The relationship of the nature of ultrasound and, in particular, its high-frequency area is hypersonic, with the structure of matter and elementary excitations in it is one of the most important features of ultrasonic waves. It allows to judge about the structure of matter based on measurements of velocity and absorption in it, depending on the frequency, as well as some external factors such as temperature, pressure, etc.

Feature of the ultrasound in the high-hypersonic ranges is the possibility of using methods of quantum mechanics, since the wavelengths and sound frequencies in these bands be of the same order with the parameters and frequencies characterizing the structure of matter.

An elastic wave of a certain frequency in this case is mapped to a quasi-particle, the photon, or quantum of sound energy. Representation of quantum mechanics is convenient when considering the various interactions in solids. For example, scattering and absorption 
of sound vibrations of the crystal lattice can be considered as the interaction of coherent and thermal phonons.

In the ultrasonic field of high intensity acoustic flows are developed, the speed of which is usually small in comparison with the oscillatory velocity of the particles $[17,18,19]$.

Flow appeared due to absorption of sound can occur in standing waves or in the boundary layer near obstacles of various types (Fig. 1)

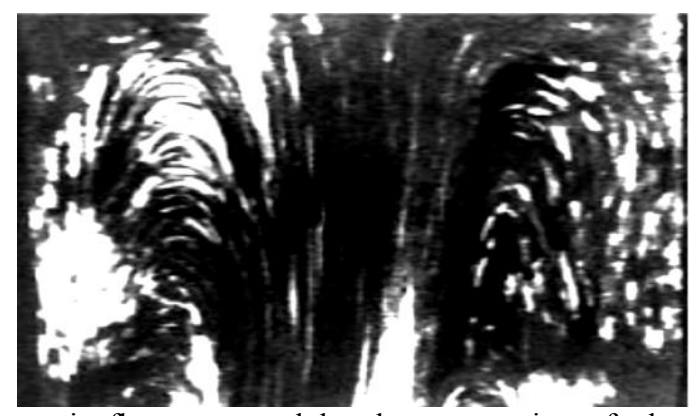

Fig. 1. Photo. The acoustic flow generated by the propagation of ultrasonic vibrations with a frequency of $5 \mathrm{MHz}$ in benzene

The radiation pressure also increases with increasing frequency, because its magnitude is proportional to the intensity of sound in the ultrasonic frequency range. It is used in the practice of acoustic measurements for determination of sound intensity.

In order to determine various effects of sound field parameters sound intensity, sound pressure, vibration velocity, the radiation pressure has reached a noticeable size, with increasing frequency requires a smaller value of the amplitude of vibrational displacement.

The most important nonlinear effect of ultrasound is the cavitation that occurs in the liquid mass of pulsating bubbles filled with steam, gas or their mixture [20]. The complex motion of bubbles and their collapse, merge with each other, etc. generate in the fluid a pulse compression (microwaves), and therefore cause local heating of the environment and ionization. These effects have an impact on the substance: the destruction of solid particles in the liquid (cavitation erosion), there is a stirring of the fluid, different physical and chemical processes start and accelerate. By changing the conditions of occurrence of cavitation, it is possible to strengthen or weaken different cavitation effects, for example, with increasing frequency of the ultrasound increases the role of micro-flows and reduced cavitation erosion, with an increase in hydrostatic pressure in the liquid, the role of microimpacts.

The increase in frequency oscillations typically leads to increased threshold intensity, corresponding to the onset of cavitation, which depends on the kind of liquid, its gas content, temperature, etc. For water in the low-frequency ultrasonic range at atmospheric pressure the intensity value is normally $0.3-1 \mathrm{~W} / \mathrm{cm}^{2}$ (Fig. 2).

Diverse applications of ultrasonic vibrations, using their various characteristics, can be divided into three areas. The first relates to obtaining information by means of ultrasonic waves, the second active impact on the substance and third - the processing and transmission of signals [21]. 


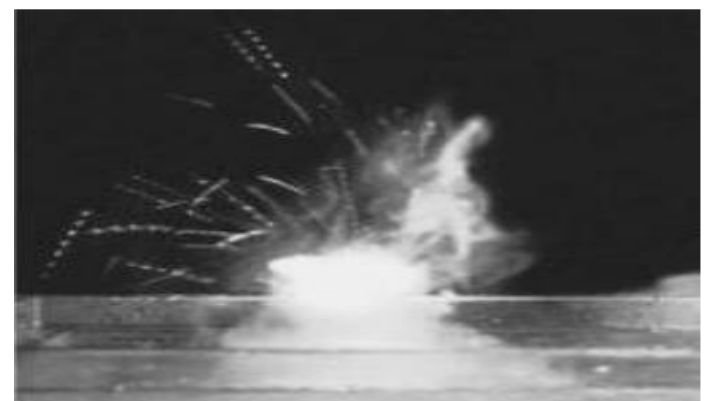

Fig. 2. Photo. The fountain of liquid that is formed when the radiation of the ultrasonic beam from the depth of liquid on its surface (the radiation frequency $1.5 \mathrm{MHz}$, intensity of $15 \mathrm{~W} / \mathrm{cm}^{2}$ ).

\section{Aim and objective}

The aim of this work is to confirm the presence of the cavitation and to identify the nature of the distribution of cavitation intensity in the liquid volume.

The objective of the work consists in the definition of cavitation according to the erosion (destruction) of the artificial barriers, and in obtaining a graphic image of the intensity of ultrasonic cavitation in the reactor volume of the experimental setup for fluid handling.

\section{Materials and methods}

There was ultrasonic reactor made of stainless steel assembled to estimate the influence of ultrasonic vibrations on water treatment with the introduction of acoustic vibrations from the bottom up, through the thickness of the liquid in the reactor volume at atmospheric pressure on the surface of the water.

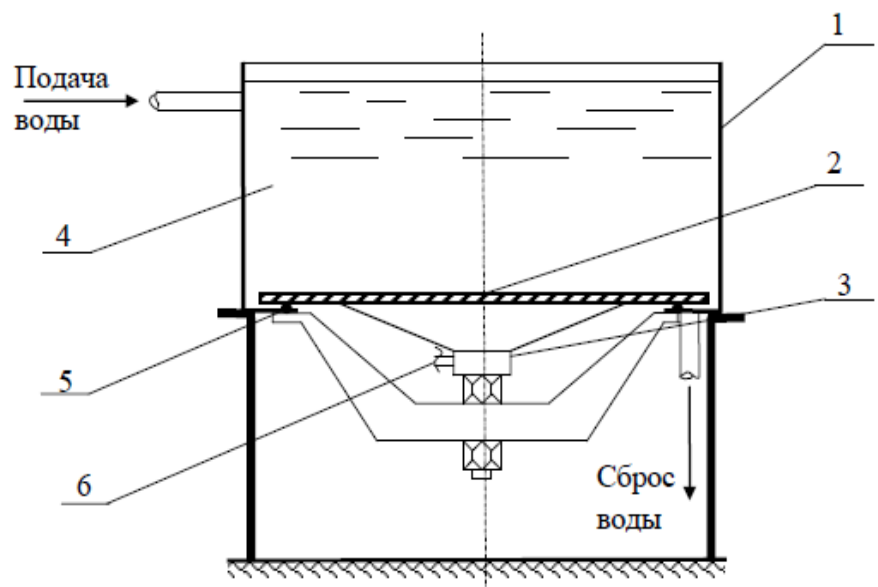

Fig. 3. Diagram of the experimental facility: 1 . The reactor of stainless steel; 2. Membrane, emitting ultrasonic waves from the bottom to the top; 3 . Magnetostrictive transducer. 4 . The treated liquid; 5 . Sealed (rubber) gaskets; 6 . Coolant.

Ultrasonic reactor of rectangular shape was equipped with magnetostrictive transducer and had the dimensions $\mathrm{LxBxH} 400 \mathrm{~mm} \times 400 \mathrm{~mm} \times 300 \mathrm{~mm}$ (the size of the membrane 300 $\mathrm{mm} \times 300 \mathrm{~mm}$ ). Converter of acoustic vibrations is located in the lower part of the reactor 
under the liquid layer. The experimental setup of the processing liquid in the ultrasonic field is presented in Fig. 3.

Cavitation is the basis of the processes of ultrasonic influence on liquid environment. Therefore, the desired accurate registration of the occurrence of cavitation phenomena. It is advisable to carry out experimental research in two stages.

\subsection{Stage 1 of the research}

It is possible to assess the collapse of cavitation bubbles in the liquid, under the action of ultrasonic vibrations according to the degree of erosion of artificial barriers (indicator). An indicator is made of foil with a size of $400 \mathrm{~mm} \times 300 \mathrm{~mm}$, and immersed in fluid, in which there is a cavitation effect.

Formed in the ultrasonic field, the bubbles collapse and create implosions, shock waves, and micro flows. Bubbles, which are formed near the surface of the foil inevitably, lead to the erosion of foil itself. In the case of a strong influence of ultrasonic vibrations, the possibility of partial or complete destruction of the leaf foil.

The intensity of the cavitation depends on the chemical composition of the liquid, the intensity of the ultrasonic field and emission of ultrasonic vibrations. In our case, the emitter and the liquid remain constant; therefore, the chemical composition of the environment, exposure of ultrasonic influence and the temperature of the sample can be changed.

Pure water and industrial wastewater of the enterprise on primary processing of wool were chosen as environments for visual detection of the cavitation.

The leaf foil size $400 \mathrm{~mm} \cdot 300 \mathrm{~mm}$, with a thickness of 15 microns in a special box that is placed in the reactor perpendicular to the radiating surface to the height of the sample.

The temperature of the source water samples was $13^{\circ} \mathrm{C}$, and the duration of ultrasonic treatment on canvas $-10 \mathrm{~min}$. To sewage, the exposure was changed from $10 \mathrm{sec}$ to 10 min, and the temperature of the water was $38^{\circ} \mathrm{C}$.

\subsection{Stage 2 of the research}

In the process of distribution of ultrasonic oscillations in the water, cavities begin to pulsate in phase with the current acoustic field. Registration of these sound effects can be done with a device that measures acoustic emission, filtering and removing the noise of the fundamental frequency. The studies determined that in the reactor under the action of ultrasonic field there are zones with different cavitation intensity and density. To identify areas of cavitation zones the device was used, allowing measuring the intensity of sound pressure when applying the ultrasonic field at any point in the reactor. This device is able to detect sound pressure, resulting from the collapse of cavitation cavities.

\section{Results}

In the first stage of experimental studies of the registration of cavitation according to the erosion of foil (the size of the canvas foil $300 \times 400 \mathrm{~mm}$.) are shown in figure 4 (a-e). The lower horizontal part of the foil was in close proximity to the emitter. Accordingly, the upper horizontal part of the fabric of the foil located on the free surface of the liquid.

Below are pictures of the actual images with traces of cavitation erosion on the canvas of foil. The resulting images presented in figure 4 , confirm the presence of cavitation regime in the liquid. 


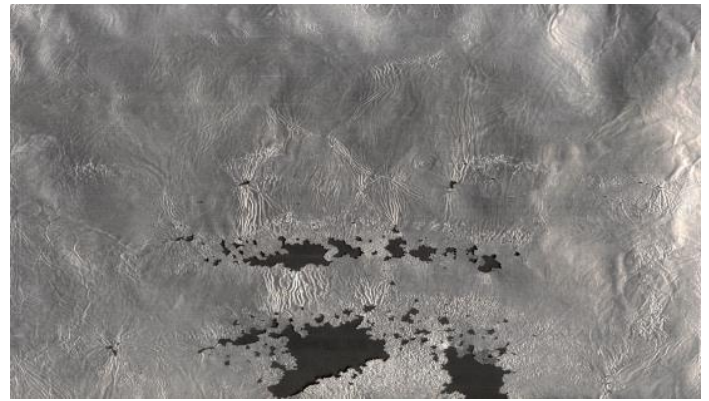

a)

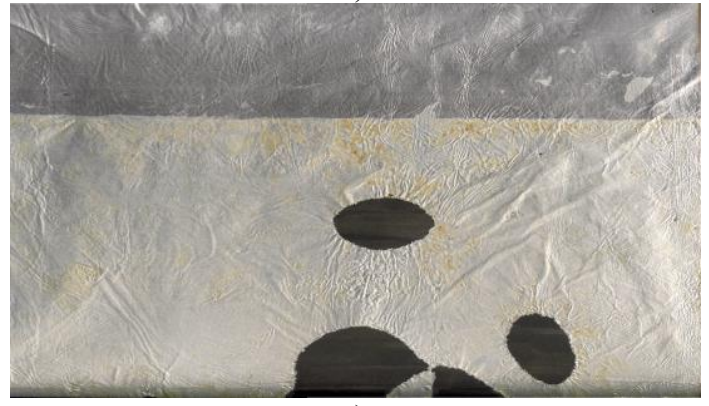

c)

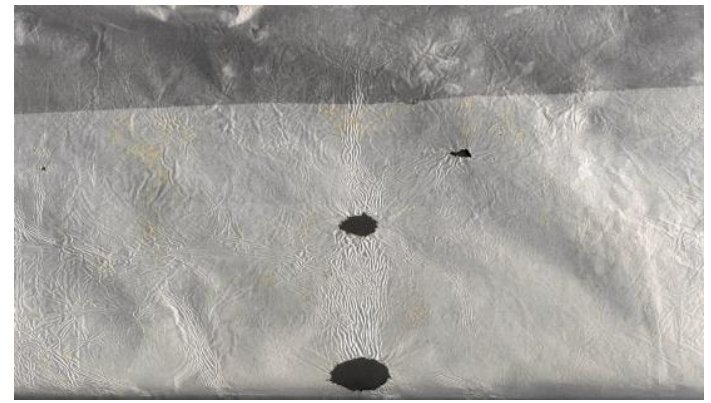

b)

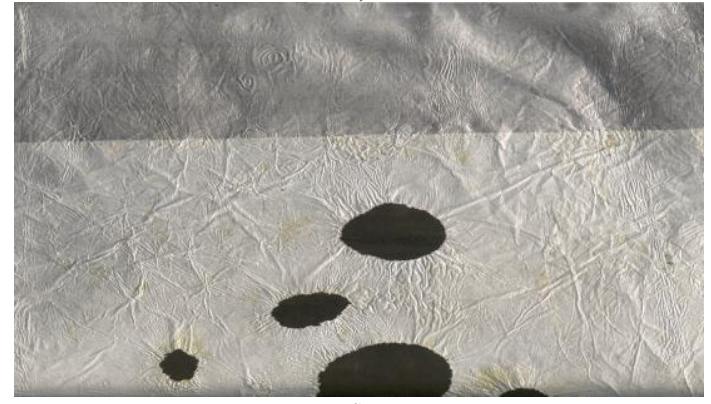

d)

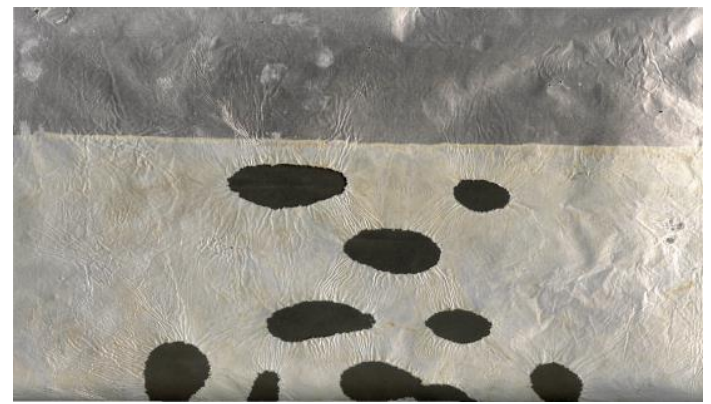

e)

Fig. 4. Photos. The results of the first stage of the experiments: a) the Time of exposure to cavitation 10 min., drinking water quality, at $\mathrm{t}=13^{\circ} \mathrm{C}$; b) the Time of exposure to cavitation for 10 seconds. industrial waste water at $\mathrm{t}=38^{\circ} \mathrm{C}$; C) the Time of exposure to cavitation $1 \mathrm{~min}$., industrial waste water, at $\mathrm{t}=38^{\circ} \mathrm{C} ; \mathrm{g}$ ) the exposure Time of cavitation $5 \mathrm{~min}$., industrial waste water, at $\mathrm{t}=38^{\circ} \mathrm{C} ; \mathrm{d}$ ) the Time of exposure to cavitation $10 \mathrm{~min}$., industrial waste water, at $\mathrm{t}=38^{\circ} \mathrm{C}$.

Cavitation destruction (erosion) was in the form of holes in the Central part of the foil. The degree of variation of physical and chemical properties of water affect the cavitation intensity in the ultrasonic field mode. Therefore, in the sample of wastewater with a high degree of variation of physical-chemical properties of water, the effect of cavitation action is superior to a similar action in water less variation of physical and chemical properties.

Thus, the resulting image on the canvas will help determine the location of foci of cavitation, and visually, with some certainty, to fix the mode of cavitation in the sample under study.

In the second stage, the obtained experimental data of the change of the sound pressure. The developed device allowed to record at selected points along the reactor cavitation pressure of the collapsing cavities. According to the obtained points to construct the plot of the isosurfaces of the distribution of sound pressure in the reactor (fig. 5). 


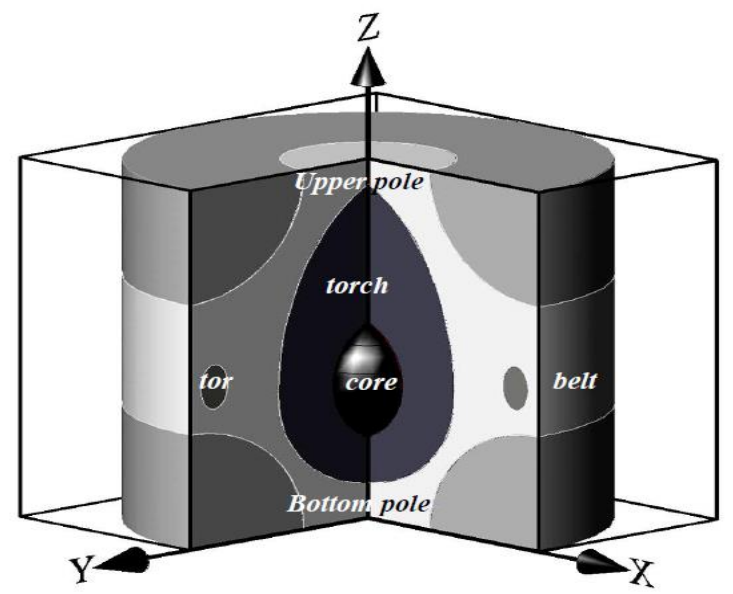

Fig. 5. Plot of the distribution of acoustic pressure in the ultrasonic reactor in the cavitation mode.

Perspective view allows witnessing the heterogeneity of areas of cavitation in the reactor volume. So, in the Central part of the plot allocated to the area of high cavitation, resembling in appearance the "torch" with a strong "core" the maximum value of acoustic pressure.

"Torch" is suspended above the radiating diaphragm and its Central part is not on the surface of the liquid medium.

The Central portion of the plot is very similar to "yule", and is characterized by an area of lower acoustic pressure than the Central part of the "torch" that corresponds to the physical parameters of the process: the length of the acoustic wave, the frequency and intensity of the radiation. However, in the Central part of the plot perimeter at the damage the "core", formed the area of the concentrated energy in the form of a "torus".

Power saturation region, the "torus" comparable area "torch", but significantly lower power of cavitation manifestations, than in the "core".

\section{Conclusions}

The first stage of experimental studies allowed obtaining real evidence of ultrasonic cavitation in the liquid medium of reactor volume.

In the second stage of experiments simultaneously installed and registered the points of maximum and minimum cavitation mode propagating in the reactor volume.

A statement of the phenomenon of cavitation in a liquid environment using the erosive characteristics of the artificial barriers is a simple and reliable method of recording the physical process. Based on a clear representation of areas of cavitation manifestations can justify the design parameters of the reactor depending on what effects you want to obtain in the liquid medium under the action of ultrasonic field in a cavitation mode.

\section{References}

1. I. P. Golyamina, Ultrasound. Little encyclopedia of Moscow (1979)

2. L. D. Rosenberg, Physics and technique of powerful ultrasound. vol. 2. (2012)

3. Vikulin, P. D., Physical and chemical manifestation of the acoustic field in the technologies of water conditioning (2004)

4. Flynn G., Physical acoustics (1967) 
5. Kardashev G. A., Physical methods of intensification of processes of chemical technology (1990)

6. Raj Baldaev, Application of ultrasound (2006)

7. N. Makisha, E3S Web of Conferences, 6, 01002 (2016)

8. Elpiner I. E., Biophysics of ultrasound (1973)

9. Margulis M. A., Adv. in phys. Sci., 170, 3, 263-287 (2000)

10. Fridman, V. M., Ultrasonic chemical equipment (1967)

11. Mironov M. A., Acoust. J., 23 (5), 825-830 (2014)

12. Leighton, T. G., The Acoustic Bubble (1994)

13. V. N. Khmelev, A. V. Shalunov, A. V. Shalunova, Ultrasonic atomization of liquids (2010)

14. N. Makisha, Procedia engineering, 165, 1087-1092 (2016)

15. Shutilov V. A., Basic physics of ultrasound (1980)

16. Kundas S. P. and others, Ultrasonic processes manufacture of electronics products (2003)

17. N. Makisha, M.Yunchina, MATEC Web of Conferences, 106, 70016 (2017)

18. Khmelev, V. N. and others, Control of the parameters of cavitating liquid media subjected to ultrasonic influence (2012)

19. Fedotkin I. M., Guly I. S., Cavitation technique and technology, their use in the industry (theory, calculations and design cavitation machines). Part 1 (1997)

20. Dezhkunov N. B., Leighton, T. G., J. of eng. Phys., 77 (1), 45-51 (2004)

21. Vikulin, P. D., Development of processes of separation of highly concentrated Chertomlyk wastewater by ultrasound (1985)

22. N. Makisha, I. Panteleeva, MATEC Web of Conferences, 106, 70015 (2017)

23. Agranat B. A., Ultrasonic technology (1974)

24. Sirotyuk M. G., Acoustic cavitation (2008)

25. Zubrilov S. P., Physico-chemical aspects of ultrasonic activation of binding solutions (1975) 